\title{
In Vivo Expression of EphrinA5-Fc in Mice Results in Cephalic Neural Crest Agenesis and Craniofacial Abnormalities
}

\author{
Hyuna Noh, Eunjeong Park, and Soochul Park*
}

\begin{abstract}
Eph receptors and their ligands ephrins have been implicated in guiding the directed migration of neural crest cells (NCCs). In this study, we found that Wnt1-Cre-mediated expression of ephrinA5-Fc along the dorsal midline of the dien- and mesencephalon resulted in severe craniofacial malformation of mouse embryo. Interestingly, expression of cephalic NCC markers decreased significantly in the frontonasal process and branchial arches 1 and 2, which are target areas for the migratory cephalic NCCs originating in the dien- and mesencephalon. In addition, these craniofacial tissues were much smaller in mutant embryos expressing ephrinA5-Fc. Importantly, EphA7-positive cephalic NCCs were absent along the dorsal dien- and mesencephalon of mutant embryos expressing ephrinA5-Fc, suggesting that the generation of cephalic NCCs is disrupted due to ephrinA5-Fc expression. NCC explant experiments suggested that ephrinA5-Fc perturbed survival of cephalic NCC precursors in the dorsal midline tissue rather than affecting their migratory capacity, which was consistent with our previous report that expression of ephrinA5-Fc in the dorsal midline is responsible for severe neuroepithelial cell apoptotic death. Taken together, our findings strongly suggest that expression of ephrinA5-Fc decreases a population of cephalic NCC precursors in the dorsal midline of the dien- and mesencephalon, thereby disrupting craniofacial development in the mouse embryos.
\end{abstract}

\section{INTRODUCTION}

Neural crests are transient cells that originate in the dorsal neuroectoderm of the neural tube. After neural crest precursors change their cell fate from epithelial to mesenchymal, they de-

Department of Biological Science, Sookmyung Women's University, Seoul 140-742, Korea

${ }^{*}$ Correspondence: scpark@sookmyung.ac.kr

Received 7 October, 2013; revised 8 November, 2013; accepted 26 November, 2013; published online 27 January, 2014

Keywords: cephalic neural crest, early brain development, EphA, ephrin-A laminate from the neuroectodermal epithelium and migrate through the underlying mesoderm (Le Douarin and Kalcheim, 1999). Eph receptors and their cognate ligands ephrins have been implicated in guiding the directed migration of neural crest cells (NCCs). Xenopus EphA4 and EphB1 are expressed in the third branchial arch, whereas ephrin-B2 is expressed in the second arch. Inhibiting these Eph receptors with a truncated receptor or ephrin-B2 overexpression results in aberrant migration of third arch NCCs into the second arch (Smith et al., 1997). In addition, mouse trunk NCCs express EphB receptors and avoid posterior somite halves expressing ephrin B ligands in vitro, suggesting that migration of trunk NCCs is restricted to anterior somite halves by repulsive interaction of ephrin-B from posterior somite halves (Wang and Anderson, 1997). These findings suggest that NCCs migrating into different target tissues may be misguided and intermingled due to loss of Eph/ ephrin repulsive signaling. More compelling evidence for the role of Eph/ephrin signaling in NCC migration comes from genetic loss-of-function studies such as those with ephrin-B1 and -B2 null mutant mice. Cephalic NCCs expressing various Eph receptors in the ephrin-B2 null mutant embryo migrate aberrantly to the clefts dividing each branchial arch where ephrin-B2 is normally expressed, but the ephrin-B2 cytoplasmic region is not essential for migration of cephalic NCCs (Adams et al., 2001). It was also reported that ephrin-B1 is expressed in migrating cephalic NCCs together with its cognate Eph receptors. Directional migration defects are observed when ephrin-B1 is eliminated only in cephalic NCCs (Davy et al., 2004). In contrast to ephrin-B2, the ephrin-B1 cytoplasmic region such as the PDZ binding domain is essential for regulating directional migration of cephalic NCCs, suggesting that reverse signaling through ephrin-B1 is critical for directional migration of cephalic NCCs.

Wnt1 plays a critical role in rostral-caudal patterning of the central nervous system (McMahon and Bradley, 1990; Thomas and Capecchi, 1990). A null mutation in Wnt1 results in agenesis of the midbrain and rostral hindbrain (Mastick et al., 1996; McMahon et al., 1992; Serbedzija et al., 1996). This developmental defect is consistent with the finding that Wnt1 is expressed along the dorsal midline of the posterior forebrain, mesencephalon, and hindbrain (Parr et al., 1993). Furthermore, Wnt1 plays a critical role in maintaining expression of the engrailed 1 (En1) transcription factor in the mesencephalon and rostral hindbrain (Danielian and McMahon, 1996). These find- 
ings strongly suggest that Wnt1 acts as an important organizer for development of the mesencephalon and rostral hindbrain. Interestingly, specific elimination of the $\beta$-catenin gene in the Wnt1 expression region results in failure of craniofacial and brain development (Haegel et al., 1995; Huelsken et al., 2000). This study further showed that null mutation of $\beta$-catenin affects NCC survival and/or differentiation, accounting for failure of craniofacial development (Brault et al., 2001). Since $\beta$-catenin is a central component in the Wnt signaling pathway, it is likely that Wnt1 acts through $\beta$-catenin to control midbrain-hindbrain development, including the formation of cephalic NCC precursors along the dorsal midline of the mesencephalon and rostral hindbrain.

EphA7 and ephrin-A5 are also co-expressed along the dorsal midline of the posterior forebrain and mesencephalon (Holmberg et al., 2000; Park et al., 2013). A previous study suggested that a truncated version of EphA7 may impair the repulsive interaction between full-length EphA7 and ephrin-A5, a potential mechanism for adhesive fusion of the cranial neural tube (Holmberg et al., 2000). However, the in vivo evidence supporting this hypothesis remains elusive. An alternative hypothesis is that trans-interaction between EphA7 and ephrin-A5 on adjacent cells in the dorsal midline triggers over-stimulation of bidirectional EphA7/ephrin-A5 signaling, which is biochemically linked with the pro-apoptotic signaling pathway (Park et al., 2013). This model was proposed as a potential mechanism for remodeling the specific brain region such as the dorsal midline of dien- and mesencephalon during early brain development (Depaepe et al., 2005; Kim et al., 2013; Park, 2013; Park et al., 2013).

In the present study, we found that overexpression of ephri$\mathrm{nA5}$-Fc along the dorsal midline of the dien- and mesencephalon resulted in severe craniofacial malformation. Evidence presented here suggests that this disruption of craniofacial development likely results from perturbation of cephalic NCC precursors originating from the dorsal midline. Together with our previous studies (Kim et al., 2013; Park, 2013; Park et al., 2013), these findings strongly suggest that cephalic NCC precursors undergo apoptotic cell death due to ephrinA5-Fc overexpression, thereby decreasing their pool in the dorsal midline of the dien- and mesencephalon, one of the important brain regions for cephalic NCC precursors.

\section{MATERIAL AND METHODS}

Mouse strains and genotyping

The eA5-eA5Fc and the EphA7-lacZ BAC transgenic lines were described previously (Park et al., 2013). The Wnt1-Cre (003829) mouse line was purchased from The Jackson Laboratory (USA).

Each BAC transgenic line was identified by polymerase chain reaction analysis of DNA extracted from tail biopsy specimens. For eA5-eA5Fc, a 360-bp sequence was amplified with primers 5'-TCATGTCTGGATCTCGACAAGGTAC-3' and 5'-GGTTGCT GCTGTTCCAGTAGAC-3'. For EphA7-LacZ, a 400-bp sequence was amplified with primers 5'- GTTACAATAAAGCAATAGC ATCACA-3' and 5'- AGGCTTCACAAATTACATTT-3'. Mouse care and breeding were performed in accordance with the institutional guidelines approved by the Sookmyung Women's University Animal Care and Use Committee.

\section{In situ hybridization and X-gal staining}

Antisense RNA probes were prepared by reverse transcription from linearized plasmids containing partial sequences of AP2
(Tfap2a) and Sox10. DNA fragments matching nt 425-676 and 1579-1740 of the mouse AP2 sequence (GenBank accession no. NM_011547.3) were used as templates for anti-sense riboprobe synthesis of Ap2; nt 484-631, 1048-1322, and 1843-1997 of the mouse Sox10 cDNA sequence (GenBank accession no. NM_011437.1) for anti-sense riboprobe synthesis of Sox10. In situ hybridization of whole mount embryos was performed as described previously (Shim et al., 2007; Yoo et al., 2011).

Embryos were fixed in $0.2 \%$ glutaraldehyde for $10 \mathrm{~min}$, washed three times in washing buffer, and stained as described previously for X-gal staining (Kim et al., 2007).

\section{Alcian Blue/Alizarin Red staining of cartilage and bone}

For the skeletal preparations, $17.5 \mathrm{dpc}$ embryos were eviscerated, skinned, and fixed in $95 \%$ ethanol for 3 days. The fixed fetuses were incubated in Alcian Blue $(150 \mathrm{mg} / \mathrm{L}$ in $0.5 \%$ acetic acid) for $12 \mathrm{~h}$ and washed in $70 \%$ ethanol for $12 \mathrm{~h}$. They were then treated with $1 \% \mathrm{KOH}$ for $6 \mathrm{~h}$, stained with Alizarin Red (50 $\mathrm{mg} / \mathrm{l}$ in $1 \% \mathrm{KOH}$ ) for $6-12 \mathrm{~h}$, cleared in $1 \% \mathrm{KOH}$, and stored in $20 \%$ glycerol (Nagy et al., 2003).

\section{Migration assay}

E9.5 embryos were dissected and placed in Hanks' balanced salt solution (GIBCO/BRL, USA) at $4^{\circ} \mathrm{C}$. Tissue containing the mesencephalon was dissected from each embryo using an Lshaped sharpened tungsten needle under a dissecting microscope. Dissected tissues were incubated in Basal Eagle's Medium (Invitrogen, USA) containing HBSS, fetal bovine serum, glucose, and glutamine. After $5 \mathrm{~h}$, the explants were treated with $1 \mu \mathrm{g}$ of $\mathrm{Fc}$ or eA5-Fc. To quantify the extent of neural crest outgrowth, digital images of the explant cultures at 5 and $22 \mathrm{~h}$ were acquired by microscopy under $10 \times$ magnification. $\mathrm{NIH}$ Image J software was used to measure the size of the explants. The migration index of the neural crest cells was obtained by dividing the outgrowth area $\left(\mathrm{mm}^{2}\right)$ with the perimeter $(\mathrm{mm})$ of the explants.

\section{RESULTS}

Cephalic NCC-derived skeletal structures are absent in mouse embryos expressing ephrinA5-Fc

In a previous report, a floxed green fluorescent protein plus ephrinA5-Fc expression cassette was specifically recombined into the ephrin-A5 bacterial artificial chromosome (BAC) (eA5eA5Fc) (Park et al., 2013) and expression of ephrinA5-Fc was induced by Wnt1-Cre, which is expressed along the dorsal midline of the dien- and mesencephalon (Dorsky et al., 1998; Ikeya et al., 1997). As all embryos expressing ephrinA5-Fc exhibited severe brain malformation but also craniofacial malformation (Park et al., 2013), we further investigated whether the development of cranial NCC-derived skeletal structures is impaired in ephrinA5-Fc expressing embryos. For this purpose, the eA5eA5Fc BAC transgenic line was crossed with the Wnt1-Cre transgenic line to generate compound transgenic embryos carrying both eA5-eA5Fc and Wnt1-Cre (ephrinA5-Fc expressing mutant embryo). Littermates carrying only eA5-eA5Fc BAC served as wild-type embryos (Figs. $1 \mathrm{~A}$ and $1 \mathrm{~B}$ ). Skeletal preparations from wild type or ephrinA5-Fc expressing mutant embryos were stained with Alizarin Red and Alician Blue (Fig. 1). Trunk skeletal structures such as the vertebral column were barely affected in the mutant embryos (Fig. 1C). However, most skeletal structures in the head region appeared to be missing in the mutant embryos (Figs. 1C and 1D). In particular, cephalic NCC-derived bone structures were prominently missing in the 


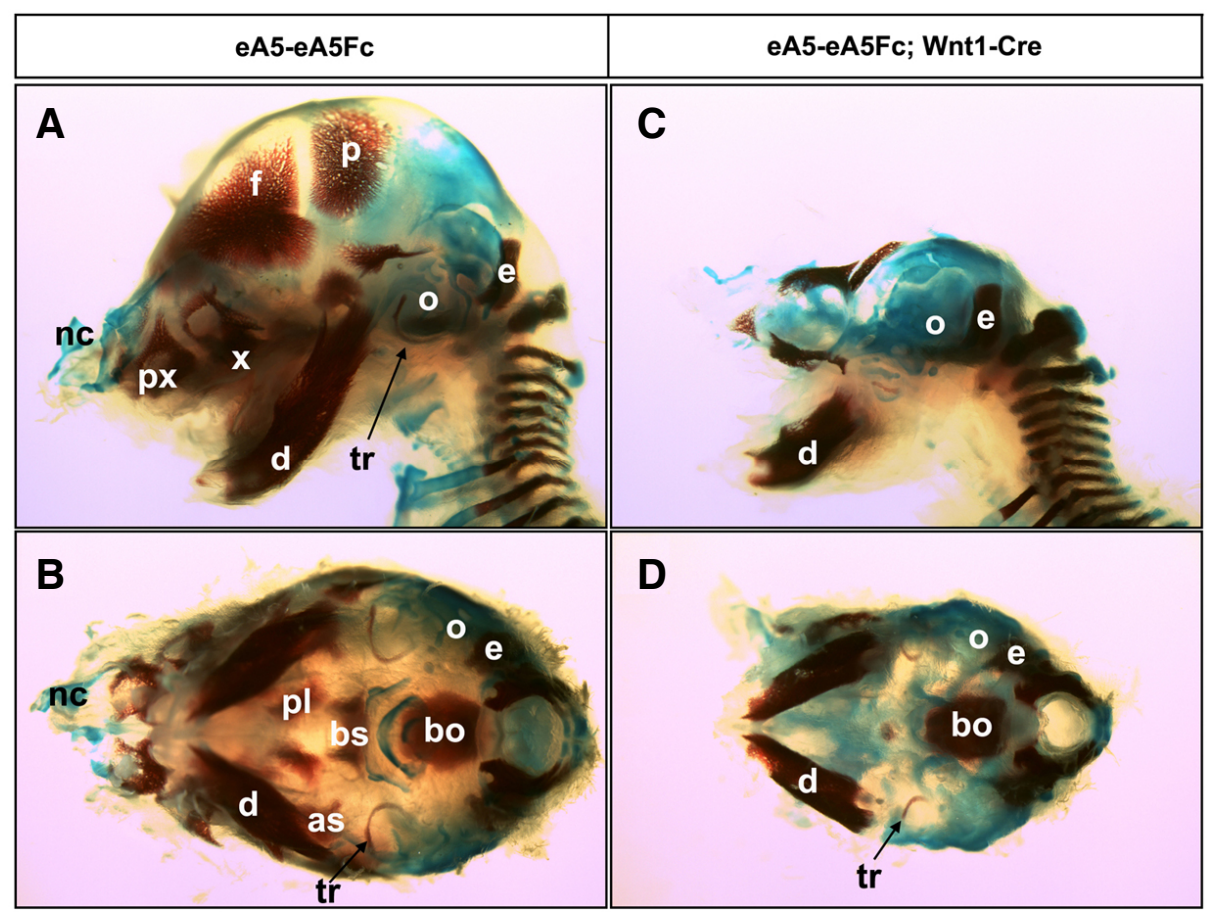

Fig. 1. Skeletal preparations of E17.5 wild type (A, B) and mutant $(C, D)$ fetuses stained with Alizarin Red and Alcian Blue; lateral (A, C) and basal $(B, D)$ views. Most of the skeletal structures in the mutant derived from the cephalic neural crest cells (NCCs) were completely lost but the mesoderm-derived skeletons were still present. Neural crest origin: $f$, frontal bone; $p$, parietal bone; $p x$, pre-maxillary bone; $\mathrm{x}$, maxilla; $\mathrm{d}$, mandible; nc, nasal capsule; pl, palatine; tr, tympanic ring. Mesoderm or neural crest origin: as, alisphenoid; bs, basisphenoid. Mesoderm origin: o, optic capsule; e, exoccipital bone; bo, basoccipital bone.

mutant embryos. These skeletal structures included the frontal bone, parietal bone, premaxilla, maxilla, mandible, nasal capsule, palatine, and the tympanic ring (Le Douarin and Kalcheim, 1999). Furthermore, alisphenoid and basisphenoid, skeletal structures derived from both cephalic NCCs and mesoderm, were also partially missing in mutant embryos. Skeletal structures that remained intact in the mutant embryos were the otic capsule, exoccipital bone, and basoccipital bone, which are derived only from mesoderm and not cephalic NCC precursors. Taken together, these findings suggest that ephrinA5-Fc expression impaired the formation of cephalic NCC-derived skeletal structures in the mutant embryos.

\section{Expression of cephalic NCC markers decreased} significantly in mutant embryos expressing ephrinA5-Fc Next, we further examined whether migratory cephalic NCCs are abundantly detected in the mutant embryo expressing ephrinA5-Fc. For this purpose, expression of two cephalic NCC markers was analyzed by whole-mount in situ RNA hybridization in E9.5 embryos. AP-2 is a transcription factor essential for survival of migratory NCCs (Mitchell et al., 1991; Schorle et al., 1996; Zhang et al., 1996). In wild-type embryos, AP2-positive cephalic NCCs were normally found in the frontonasal process, mesencephalon, rhombomere (r) 2 and 4, and branchial arches 1 and 2, whereas the number of AP2-positive cells decreased significantly in mutant embryos expressing ephrinA5-Fc, particularly in the frontonasal process and mesencephalon (Figs. $2 \mathrm{~A}$ and $2 \mathrm{C}$ ). However, migratory cephalic NCCs extending from r2 and $\mathrm{r} 4$ into the branchial arches appeared to be present in the mutant embryos, although their staining intensity decreased to some degree (Fig. 2C). Sox10 is another NCC marker important for peripheral nervous system development (Paratore et al., 2001). Sox10 was normally expressed in the frontonasal process, cranial ganglia, and branchial arches 1 and 2 in the cranial region of wild-type embryos, whereas Sox10 was barely expressed in the corresponding regions of mutant embryos expressing ephrinA5-Fc (Figs. 2B and 2D). Importantly, a smaller sized dien- and mesencephalon was consistently observed in mutant embryos expressing ephrinA5-Fc (Figs. 2C and 2D). Furthermore, the dorsal midline of the dien- and mesencephalon in mutant embryos was also absent (Figs. 2C and 2D) (Park et al., 2013). Because the dorsal midline of the dien- and mesencephalon is a region for birth of cephalic NCC precursors, it is likely that the frontonasal process and branchial arches were absent or much smaller in mutant embryos due to agenesis of cephalic NCCs from the dorsal midline. Taken together, these results suggest that expression of ephrinA5-Fc perturbed generation of cephalic NCCs in the dorsal midline of mutant embryos.

\section{Agenesis of EphA7-positive cephalic NCCs in mutant embryos expressing ephrinA5-Fc}

Many precursors for the head skeleton originate from cephalic NCCs that delaminate from the dorsal neural tube, where Wnt1 gene is expressed (Dorsky et al., 1998; Ikeya et al., 1997). Those cells migrate along segmental pathways to the frontonasal mass and branchial arches. Interestingly, EphA7 is co-expressed with ephrin-A5 along the dorsal midline of the dien- and mesencephalon (Park et al., 2013) but is also detected in the branchial arches and frontonasal process (Fig. 3A). Therefore, we postulated that an interaction between EphA7 and ephrinA5 may be involved in generating cephalic NCCs in the dorsal midline and that EphA7-expressing cephalic NCCs migrate from the dorsal midline to the frontonasal process and branchial arches. However, ectopic expression of ephrinA5-Fc may perturb generation of EphA7-positive cephalic NCCs in the dorsal midline. Compound transgenic embryos carrying eA5-eA5Fc BAC, Wnt1-Cre, and EphA7-LacZ BAC were generated to 

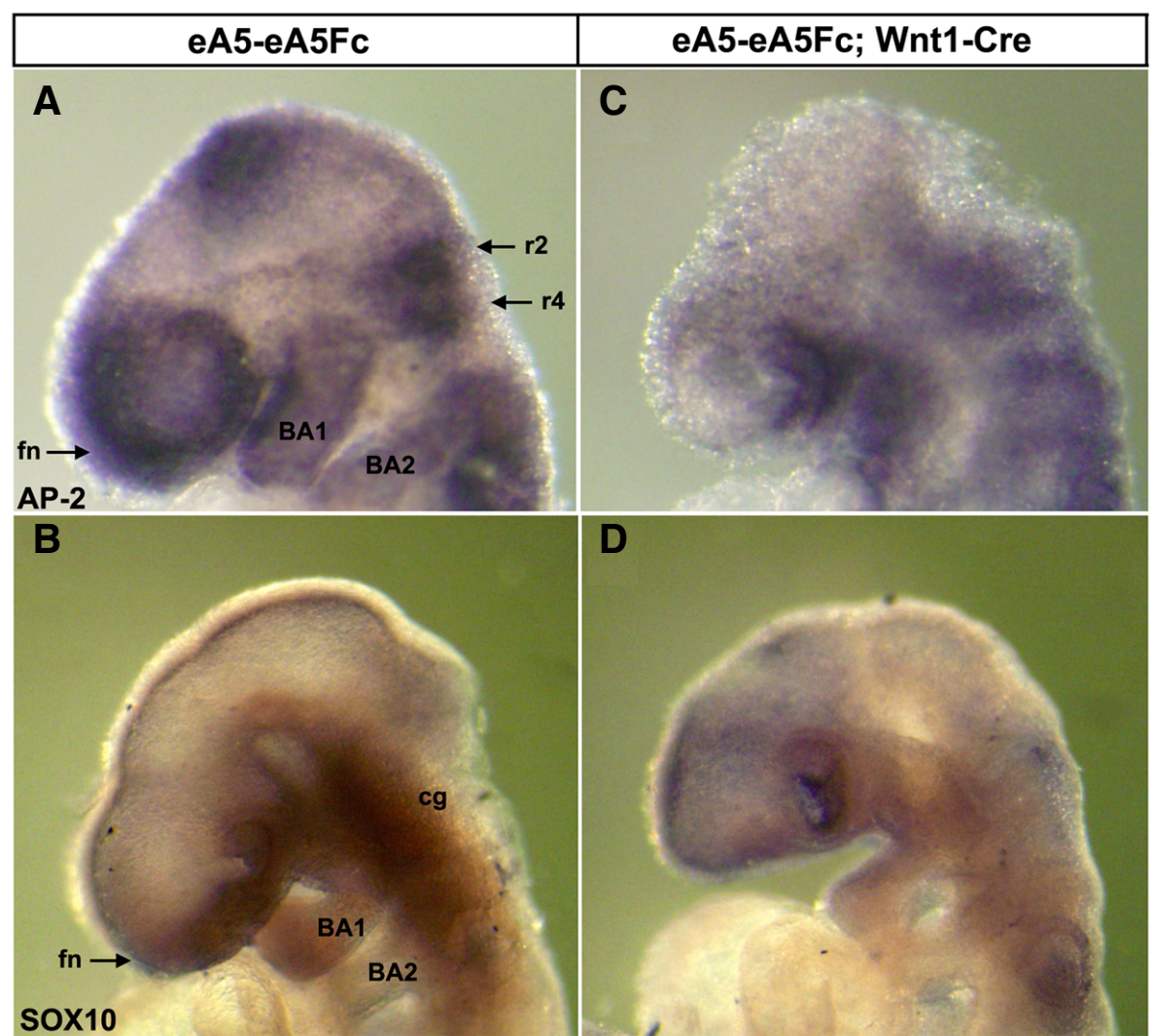

Fig. 2. Whole-mount in situ hybridization analysis of E9.5 wild type (A, B) vs. mutant (C, D) embryos using neural crest cell (NCC)specific probes. Note that the AP-2 expressing region was prominently reduced in the frontonasal process (fn), dien- and mesencephalon, and rhombomeres 2 and 4 of the mutant (C) compared with those in the wild type (A). Expression of SOX10 in the frontonasal process, cranial ganglion (cg), and branchial arches 1 and 2 [BA1, BA2 in (B)] was also reduced in mutants (D). fn, frontonasal process; BA1, branchial arch 1; BA2, branchial arch 2; r2, rhombomere 2; r4, rhombomere 4; cg, cranial ganglions.

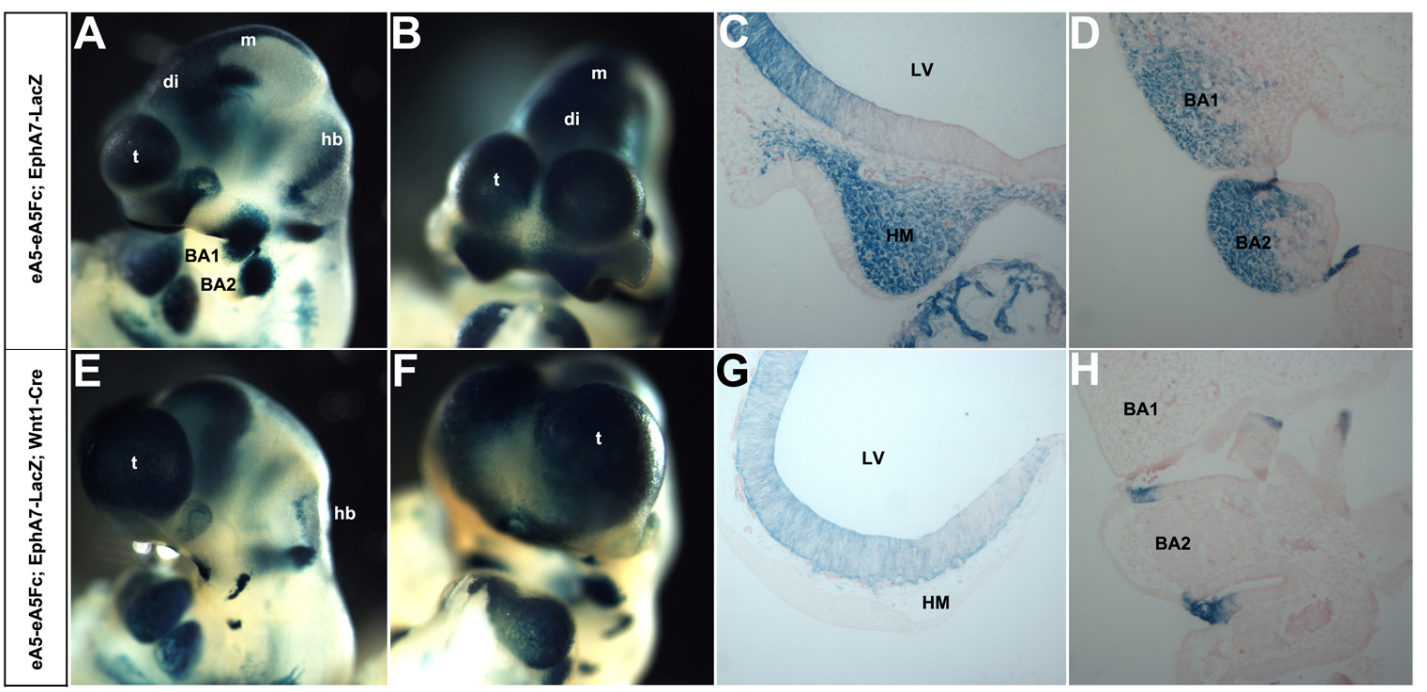

Fig. 3. Analysis of EphA7-expressing cephalic neural crest cells (NCCs) of E9.5 wild type (A, B) vs. mutant (E, F) embryos using whole mount $X$-gal staining. (A, E) Lateral views of E10.5 embryos after whole-mount X-gal staining. (B, F) Frontal views of E10.5 embryos shown in (A) and $(E)$, respectively. $(C, G)$ Coronal sections of the embryo shown in $(A)$ and $(E)$. EphA7-expressing cephalic NCCs are missing in the head mesenchymal tissue of the mutant. (D, H) Coronal sections of the embryo shown in (A) and (E). EphA7-expressing cephalic NCCs are also missing in BA1 and BA2 regions of the mutant. BA1, branchial arch 1; BA2, branchial arch 2; di, diencephalon; hb, hindbrain; LV, lateral ventricle; $\mathrm{m}$, mesencephalon; $\mathrm{HM}$, head mesenchyme; t, telencephalon. 
A

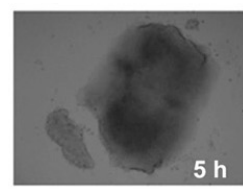

Fc

B

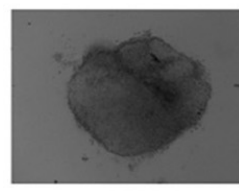

eA5-Fc
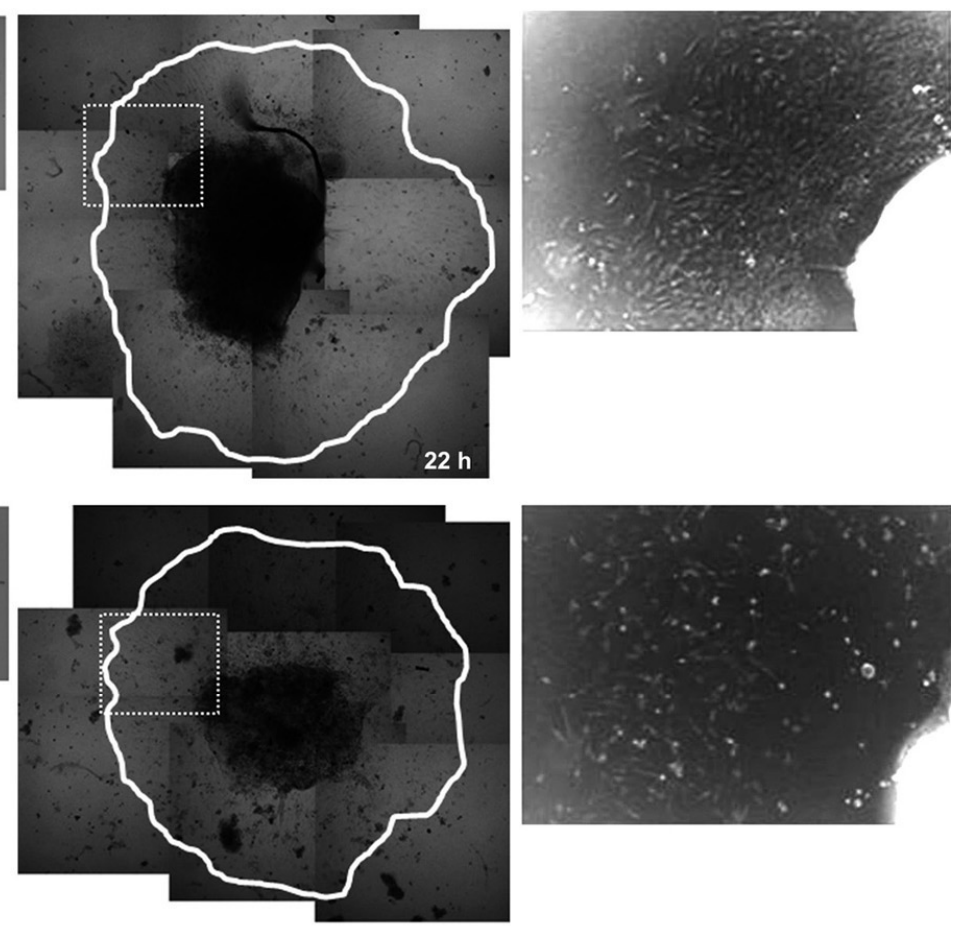

Fig. 4. EphrinA5-Fc treatment does not affect emigration of neural crest cells but reduce cell densities. (A, B) E9.5 wild type neural tubes were isolated and plated into cell culture dishes. After the explants were settled for $5 \mathrm{~h}$, the explants were treated with $\mathrm{Fc}$ alone or ephrinA5-Fc. Then, the migration index was measured to compare their outgrowth area. Note that the neural tube explants trea-ted with ephrinA5-Fc show lower cell density in its core area $(B$, third panel). (C) Quantification of in vitro outgrowth from the neural tube did not reveal significant differences in emigration of neural crest cells (NCCs) irrespective of ephrinA5Fc treatment $(p>0.5)$.

C

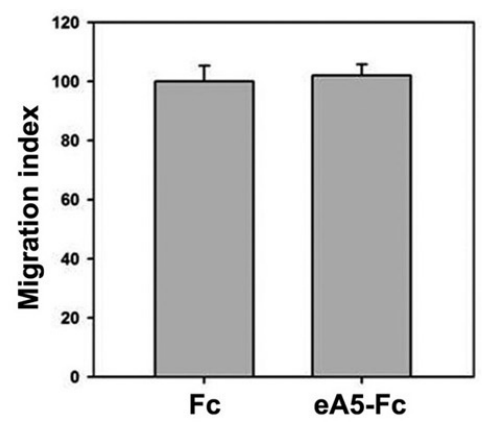

better visualize those cephalic NCCs expressing EphA7 (Figs. $3 E$ and $3 F$ ), whereas littermates carrying both eA5-eA5FC and EphA7-LacZ BACs served as wild-type embryos (Figs. 3A and 3B). BAC transgenic embryos containing EphA7-LacZ recapitulate a well established expression pattern of endogenous EphA7 (Figs. 3A and 3B) (Kim and Park, 2011). Whole mount X-gal staining of these compound transgenic embryos allowed us to analyze craniofacial structures in more detail. First, the dorsal midline structure of the dien- and mesencephalon was completely absent in mutant embryos expressing ephrinA5-Fc (Figs. $3 \mathrm{E}$ and $3 \mathrm{~F}$ ). This result is consistent with our previous finding that ectopic expression of ephrinA5-Fc in the dorsal midline results in a dramatic increase in apoptotic cell death of neuroepithelial cells expressing EphA7 (Park et al., 2013). Second, the frontonasal mass containing EphA7-positive migratory cephalic NCCs was almost eliminated in the mutant embryo (Fig. 3G). This result was not surprising because most cells in the frontonasal region are derived from cephalic NCCs originating from the dien- and mesencephalon. Third, branchial arch 1 containing EphA7-positive migratory cephalic NCCs decreased significantly in size (Figs. 3E, 3F, and $3 \mathrm{H}$ ). This result was also con- sistent with previous studies showing that the anterior half of branchial arch 1 is derived from the posterior mesencephalon and rhombomere 1 (Le Douarin and Kalcheim, 1999). Branchial arch 2 was also smaller but much less affected compared with branchial arch 1 (Fig. $3 \mathrm{H}$ ). Taken together, these results strongly suggest that generation of EphA7-positive cephalic NCCs in the dorsal midline of the dien- and mesencephalon was defective in mutant embryos expressing ephrinA5-Fc, accountting for malformation of craniofacial structures.

To further investigate the migratory ability of cephalic NCCs, cephalic NCC explants corresponding to the dorsal part of the mesencephalon were prepared from wild-type embryos at E9.5. After the explants were settled in the culture dish for $5 \mathrm{hs}$, the explants were further treated with $\mathrm{Fc}$ alone or ephrinA5-Fc, and the extent of NCC outgrowth was analyzed $17 \mathrm{~h}$ after adding the ligand. However, measuring the outgrowth area as indicated by the migration index (Fuchs et al., 2009) did not reveal significant differences between the $\mathrm{Fc}$ and ephrinA5-Fc treated groups (Fig. 4B second panel; Fig. 4C), although the overall cell densities in the NCC outgrowth area decreased in the ephrinA5-Fc-treated group (Fig. 4B, third panel). Importantly, the neural 
tube explants treated with ephrinA5-Fc revealed much lower cell density in its core region (Fig. $4 \mathrm{~B}$, second panel). These results suggest that ephrinA5-Fc perturbs the survival of cephalic NCC precursors in the dorsal midline tissue rather than affecting their migratory capacity.

\section{DISCUSSION}

In this study, we found that expression of ephrinA5-Fc disrupted the formation of cephalic NCCs in the dorsal midline of the dien- and mesencephalon. This perturbation of cephalic NCC precursors is likely to be a major cause for the dramatic craniofacial malformation of mutant embryos expressing ephrinA5-Fc.

An important issue is how expression of ephrinA5-Fc impairs the formation of cephalic NCCs in the developing brain. EphA7 is co-expressed with ephrin-A5 along the dorsal midline of the dien- and mesencephalon (Park et al., 2013). Because ephri$\mathrm{nA5}-\mathrm{Fc}$ was on the ephrin-A5 BAC and its expression was induced by Wnt1-Cre, it is expected that ephrinA5-Fc was secreted from cells expressing endogenous ephrin-A5 and then bound to EphA7 in the adjacent cells. Previous studies also indicated that Eph receptors and their ephrin ligands are segregated into distinct membrane regions within the same cell (Marquardt et al., 2005). This arrangement of Eph receptors and ephrins within the same cell allows them to interact in a trans-configuration with their counterparts in the adjacent cells. Therefore, it is predicted that the secreted ephrinA5-Fc is bound to EphA7 in adjacent cells and that this trans-interaction increases forward signaling downstream of the EphA7 receptor. More importantly, expression of ephrinA5-Fc in the dorsal midline was responsible for severe neuroepithelial cell apoptotic death, revealing that Eph/ephrin signaling is biochemically linked with pro-apoptotic signaling. Furthermore, we found that EphA7-expressing cells in the dorsal midline were selectively eliminated when expression of ephrinA5-Fc was induced by Wnt1-Cre (Park et al., 2013). Therefore, it is likely that apoptotic cell death is a major cause for agenesis of cephalic NCC precursors and craniofacial malformation in mutant embryos.

Notably, mutant embryos expressing ephrinA5-Fc displayed a phenotype similar to what was observed for the Wnt1 or $\beta$ catenin null mutant embryo (Brault et al., 2001; Ikeya et al., 1997). Null mutation of Wnt1 resulted in failure of the midbrain, and specific inactivation of the $\beta$-catenin gene in the domain of Wnt1 expression also resulted in dramatic brain malformation and no discernible midbrain. It remains to be determined why expression of ephrinA5-Fc results in failure of midbrain development. One possible hypothesis is that EphA7 is genetically downstream of Wnt $1 / \beta$-catenin signaling. For example, Wnt $1 / \beta-$ catenin signaling may turn on EphA7 gene expression in the dorsal midline and then the EphA7 receptor is localized to the cell surface and involved in initiating emigration of cephalic NCC precursors through interaction with ephrin-A5, whereas over-stimulation of EphA7-mediated forward signaling may be lethal for cell survival and provide a potential mechanism for controlling progenitor population size and tissue remodeling. In this respect, a genetic link between gene expression of EphA7 and Wnt $1 / \beta$-catenin signaling would facilitate the formation of cephalic NCC precursors to be understood at the molecular level.

\section{ACKNOWLEDGMENTS}

This study was supported by Sookmyung Women's University Research Grants (1-1303-0091).

\section{REFERENCES}

Adams, R.H., Diella, F., Hennig, S., Helmbacher, F., Deutsch, U., and Klein, R. (2001). The cytoplasmic domain of the ligand ephrinB2 is required for vascular morphogenesis but not cranial neural crest migration. Cell 104, 57-69.

Brault, V., Moore, R., Kutsch, S., Ishibashi, M., Rowitch, D.H., McMahon, A.P., Sommer, L., Boussadia, O., and Kemler, R. (2001). Inactivation of the beta-catenin gene by Wnt1-Cre-mediated deletion results in dramatic brain malformation and failure of craniofacial development. Development 128, 1253-1264.

Danielian, P.S., and McMahon, A.P. (1996). Engrailed-1 as a target of the Wnt-1 signalling pathway in vertebrate midbrain development. Nature 383, 332-334.

Davy, A., Aubin, J., and Soriano, P. (2004). Ephrin-B1 forward and reverse signaling are required during mouse development. Genes Dev. 18, 572-583.

Depaepe, V., Suarez-Gonzalez, N., Dufour, A., Passante, L., Gorski, J.A., Jones, K.R., Ledent, C., and Vanderhaeghen, P. (2005). Ephrin signalling controls brain size by regulating apoptosis of neural progenitors. Nature 435, 1244-1250.

Dorsky, R.I., Moon, R.T., and Raible, D.W. (1998). Control of neural crest cell fate by the Wnt signalling pathway. Nature 396, 370373.

Fuchs, S., Herzog, D., Sumara, G., Buchmann-Moller, S., Civenni, G., Wu, X., Chrostek-Grashoff, A., Suter, U., Ricci, R., Relvas, J.B., et al. (2009). Stage-specific control of neural crest stem cell proliferation by the small rho GTPases Cdc42 and Rac1. Cell Stem Cell 4, 236-247.

Haegel, H., Larue, L., Ohsugi, M., Fedorov, L., Herrenknecht, K., and Kemler, R. (1995). Lack of beta-catenin affects mouse development at gastrulation. Development 121, 3529-3537.

Holmberg, J., Clarke, D.L., and Frisen, J. (2000). Regulation of repulsion versus adhesion by different splice forms of an Eph receptor. Nature 408, 203-206.

Huelsken, J., Vogel, R., Brinkmann, V., Erdmann, B., Birchmeier, C., and Birchmeier, W. (2000). Requirement for beta-catenin in anterior-posterior axis formation in mice. J. Cell Biol. 148, 567-578.

Ikeya, M., Lee, S.M., Johnson, J.E., McMahon, A.P., and Takada, S. (1997). Wnt signalling required for expansion of neural crest and CNS progenitors. Nature 389, 966-970.

Kim, Y., and Park, S. (2011). Identification of EphA7 BAC clone containing a long-range dorsal midline-specific enhancer. BMB Rep. 44, 113-117.

Kim, Y., Song, E., Choi, S., and Park, S. (2007). Engineering lacZ Reporter gene into an ephA8 bacterial artificial chromosome using a highly efficient bacterial recombination system. J. Biochem. Mol. Biol. 40, 656-661.

Kim, Y., Park, E., Noh, H., and Park, S. (2013). Expression of EphA8Fc in transgenic mouse embryos induces apoptosis of neural epithelial cells during brain development. Dev. Neurobiol. 73, 702-712.

Le Douarin, N., and Kalcheim, C. (1999). The Neural Crest (Cambridge University Press).

Marquardt, T., Shirasaki, R., Ghosh, S., Andrews, S.E., Carter, N., Hunter, T., and Pfaff, S.L. (2005) Coexpressed EphA receptors and ephrin-A ligands mediate opposing actions on growth cone navigation from distinct membrane domains. Cell 121, 127-139.

Mastick, G.S., Fan, C.M., Tessier-Lavigne, M., Serbedzija, G.N., McMahon, A.P., and Easter, S.S., Jr. (1996). Early deletion of neuromeres in Wnt-1-/- mutant mice: evaluation by morphological and molecular markers. J. Comp. Neurol. 374, 246-258.

McMahon, A.P., and Bradley, A. (1990). The Wnt-1 (int-1) protooncogene is required for development of a large region of the mouse brain. Cell 62, 1073-1085.

McMahon, A.P., Joyner, A.L., Bradley, A., and McMahon, J.A. (1992). The midbrain-hindbrain phenotype of Wnt-1-Wnt-1- mice results from stepwise deletion of engrailed-expressing cells by 9.5 days postcoitum. Cell 69, 581-595.

Mitchell, P.J., Timmons, P.M., Hebert, J.M., Rigby, P.W., and Tjian, R. (1991). Transcription factor AP-2 is expressed in neural crest cell lineages during mouse embryogenesis. Genes Dev. 5, 105119.

Nagy, A., Gertsenstein, M., Vintersten, K., and Behringer, R. (2003). Manipulating the Mouse Embryo: A Laboratory Manual. Cold 
Spring Harbor; (NY: Cold Spring Harbor Laboratory Press).

Paratore, C., Goerich, D.E., Suter, U., Wegner, M., and Sommer, L. (2001). Survival and glial fate acquisition of neural crest cells are regulated by an interplay between the transcription factor Sox 10 and extrinsic combinatorial signaling. Development 128, 39493961.

Park, S. (2013). Brain-region specific apoptosis triggered by Eph/ ephrin signaling. Exp. Neurobiol. 22, 143-148.

Park, E., Kim, Y., Noh, H., Lee, H., Yoo, S., and Park, S. (2013). EphA/ephrin-A signaling is critically involved in region-specific apoptosis during early brain development. Cell Death Differ. 20, 169-180.

Parr, B.A., Shea, M.J., Vassileva, G., and McMahon, A.P. (1993). Mouse Wnt genes exhibit discrete domains of expression in the early embryonic CNS and limb buds. Development 119, 247261.

Schorle, H., Meier, P., Buchert, M., Jaenisch, R., and Mitchell, P.J. (1996). Transcription factor AP-2 essential for cranial closure and craniofacial development. Nature 381, 235-238.

Serbedzija, G.N., Dickinson, M., and McMahon, A.P. (1996). Cell death in the CNS of the Wnt-1 mutant mouse. J. Neurobiol. 31, 275-282.

Shim, S., Kim, Y., Shin, J., Kim, J., and Park, S. (2007). Regulation of EphA8 gene expression by TALE homeobox transcription factors during development of the mesencephalon. Mol. Cell. Biol. 27, 1614-1630.

Smith, A., Robinson, V., Patel, K., and Wilkinson, D.G. (1997). The EphA4 and EphB1 receptor tyrosine kinases and ephrin-B2 ligand regulate targeted migration of branchial neural crest cells. Curr. Biol. 7, 561-570.

Thomas, K.R., and Capecchi, M.R. (1990). Targeted disruption of the murine int-1 proto-oncogene resulting in severe abnormalities in midbrain and cerebellar development. Nature 346, 847 850.

Wang, H.U., and Anderson, D.J. (1997). Eph family transmembrane ligands can mediate repulsive guidance of trunk neural crest migration and motor axon outgrowth. Neuron 18, 383-396.

Yoo, S., Kim, Y., Noh, H., Lee, H., Park, E., and Park, S. (2011) Endocytosis of EphA receptors is essential for the proper development of the retinocollicular topographic map. EMBO J. 30, 1593-1607.

Zhang, J., Hagopian-Donaldson, S., Serbedzija, G., Elsemore, J., Plehn-Dujowich, D., McMahon, A.P., Flavell, R.A., and Williams, T. (1996). Neural tube, skeletal and body wall defects in mice lacking transcription factor AP-2. Nature 381, 238-241. 